\title{
Insulin signaling pathway in the masseter muscle of dexamethasone-treated rats
}

\author{
IGOR RABELO DE FRANÇA ${ }^{1}$, DANIELA MENESES-SANTOS ${ }^{2}$, \\ GABRIELA VIRGINIA MOREIRA ${ }^{3}$, FÁBIO BESSA LIMA ${ }^{3}$, \\ CARLA ROBERTA DE OLIVEIRA CARVALHO ${ }^{3}$, ANDERSON CARLOS MARÇAL ${ }^{2, *}$
}

\author{
${ }^{1}$ Department of Medicine, Center of Biological Sciences and Health, Federal University of Sergipe, Aracaju, Brazil \\ ${ }^{2}$ Department of Morphology, Center of Biological Sciences and Health, Federal University of Sergipe, São Cristóvão, Brazil \\ ${ }^{3}$ Department of Physiology and Biophysics, Institute of Biomedical Sciences, University of São Paulo, São Paulo, Brazil \\ ${ }^{*}$ Corresponding author: Anderson Carlos Marçal; Centro de Ciências Biológicas e da Saúde, Universidade Federal de Sergipe, Cidade Universitária \\ Prof. José Aloísio de Campos, Jardim Rosa Elze, CEP: 49100-000, São Cristóvão, Sergipe, Brazil; Phone: +55 7921056622 ; \\ E-mail: acmarcal@yahoo.com.br
}

(Received: December 8, 2017; Accepted: July 20, 2018)

\begin{abstract}
Background and aims: The treatment with glucocorticoids may induce molecular changes in the level and/or degree of phosphorylation of proteins located downstream of the insulin receptor/insulin-like growth factor receptor (IR/IGFIR) in many tissues. However, few studies have investigated the intracellular insulin pathway in the masseter muscle. Therefore, this study aimed to analyze the IR/IGFIR signaling pathway in the masseter muscle of rats treated with dexamethasone. Materials and methods: Male Wistar rats were divided into two groups: control group (intraperitoneally injected with $0.9 \% \mathrm{NaCl}$ solution) and dexamethasone group [intraperitoneally injected with $1 \mathrm{mg} / \mathrm{kg}$ (bw) dexamethasone solution] for 10 consecutive days. Sections of the masseter muscle were removed at time zero and after the infusion of regular insulin into the portal vein. Results: Dexamethasone administration induces body weight loss without changing masseter muscle weight and reduces the expression of total IR and PI3K proteins; total levels of IRS1, Akt, and ERKI remain unchanged between groups. The degree of phosphorylation/activity of IRS1 after insulin stimulus increased only in the control group; degree of phosphorylation of Akt increased in both groups, but this increase was attenuated in the dexamethasone group. Discussion and conclusion: The degree of phosphorylation/activity in the masseter muscle is different from that in other muscle territories.
\end{abstract}

Keywords: glucocorticoids, mastication, masseter muscle, diabetes mellitus, skeletal muscle

\section{Introduction}

Changes in lifestyle in recent decades have altered the metabolic profile of the world's population $[1,2]$. The increase in the number of overweight or obese individuals as a result of excessive consumption of hypercaloric foods, sedentarism, and low intake of fibers has contributed to the morbimortality of populations, with an increased prevalence of diabetes mellitus type 2 , high blood pressure, dyslipidemia, peripheral resistance to insulin, and other disorders that characterize metabolic syndrome [ 1 , 3-5].

Among these metabolic changes, resistance to insulin is a clinical condition characterized by a decreased response to the action of insulin in target organs, which results in high serum levels of glucose associated with compensatory hyperinsulinemia through an increase in the number of beta-pancreatic cells [6-9].

Resistance to insulin in peripheral tissues (adipose tissue, liver, and skeletal muscle) is partly caused by molecular adjustments in the level and/or degree of phosphorylation of proteins involved in the intracellular insulin receptor/insulin-like growth factor receptor (IR/IGF1R) signaling pathway [10-15].

Proteins located downstream of the IR/IGFl signaling pathway can be phosphorylated at tyrosine residues of IR substrates (IRSs), mainly IRSI and IRS2. IRS1 is involved in the stimulation of glucose uptake and glycogen storage

This is an open-access article distributed under the terms of the Creative Commons Attribution-NonCommercial 4.0 International License, which permits unrestricted use, distribution, and reproduction in any medium for non-commercial purposes, provided the original author and source are credited, a link to the CC License is provided, and changes - if any - are indicated. 
in the skeletal muscle, whereas IRS2 acts on the metabolism of the skeletal muscle, adipose tissue, and liver $[16,17]$. The phosphorylation of these substrates leads to the activation of several kinases, namely phosphatidylinositol 3-kinase (PI3K), which causes the activation of protein kinase $\mathrm{B}$ ( $\mathrm{PKB}$ or $\mathrm{Akt}$ ) through phosphorylation at serine residues. Akt has several roles, such as glucose transportation, glycogen synthesis, protein synthesis, lipogenesis, and liver gluconeogenesis inhibition $[8,15]$.

Dexamethasone, which is a potent synthetic glucocorticoid with dose-dependent anti-inflammatory and immunosuppressant activities, has been used to investigate intracellular insulin signaling pathways during resistance to insulin $[7,18,19]$. In addition to resistance to insulin, the adverse effects of its chronic use include dyslipidemia, increased localized fat, and muscle deterioration, which are manifestations that are similar to those in Cushing's syndrome [20-24].

Glucocorticoids can decrease the expression of IRS1, PI3K, and PKB, causing the migration of a reduced amount of GLUT4 (characteristically found in the skeletal muscle) toward the membrane, thus decreasing glucose uptake and glycogen synthesis $[15,25,26]$.

Among the tissues that respond to insulin, the skeletal muscle accounts for approximately $75 \%$ of glucose uptake as it is the largest muscle in the human body [14]. Various skeletal muscle territories, such as the tibialis anterior, extensor digitorum longus [22], soleus, epitrochleoanconeus [27, 28], and gastrocnemius [29] muscles, have been used as experimental models to assess the intracellular insulin signaling pathway under different metabolic conditions [30]. However, this pathway has not yet been fully understood in the masseter muscle. Therefore, this study aimed to analyze IR/IGFl/IRSl/Akt/PI3K in the masseter muscle under normal conditions and in the presence of resistance to insulin induced by dexamethasone.

\section{Materials and Methods}

\section{Rat model}

Animal procedures were conducted according to the guidelines of the Ethics Committee on Animal Research of the Federal University of Sergipe, which approved the study under protocol (CEPA 54/2009). Male Wistar rats were obtained from the central animal house of the Federal University of Sergipe, São Cristóvão, Sergipe, Brazil, and they weighed between 250 and $300 \mathrm{~g}$. The rats were kept in a controlled environment (temperature of $23 \pm 2{ }^{\circ} \mathrm{C}$ and 12 -h light-dark cycle of period between 6:00 [zeitgeber time (ZT0) and 18:00 ZT12] and the rodents were offered commercial feed (Ração Labina, São Paulo, SP) and water ad libitum. The rats were randomly divided into two groups: control group (CON group), intraperitoneally injected with $0.9 \% \quad \mathrm{NaCl}$ (saline solution), and dexamethasone group (DEX group), intraperitoneally injected with $1 \mathrm{mg} / \mathrm{kg}$ body weight of dexamethasone (Decadron ${ }^{\circledR}$ Aché, Guarulhos-SP, Brazil) for 10 consecutive days during the ZT6 period (12:00). The body weight of the rats was measured on a daily basis. On the 1lth day, the rats were euthanized with the anesthetic sodium thiopental (Thiopentax ${ }^{\circledR}$, Cristália, Itapira-SP, Brazil) (body weight: $40 \mathrm{mg} / \mathrm{kg}$ ). Euthanasia was performed on a second experimental group of rats, and their body weight and masseter muscle weight were measured; then, the mastication (masseter) muscles were desiderated to obtain muscle dry weight $\left(48 \mathrm{~h}, 50{ }^{\circ} \mathrm{C}\right)$, for subsequent statistical analyses.

\section{Protein quantification by Western blotting}

Sections of the right masseter muscle were isolated to establish time zero (basal protein phosphorylation). Subsequently, $0.5 \mathrm{ml}$ of saline solution containing $5 \mathrm{mmol} / \mathrm{L}$ of insulin per $\mathrm{kg}$ of body weight was injected into the portal vein, and after $3 \mathrm{~min}$, the sections of the left masseter muscle were removed from all the rats in both groups. The sections were macerated and transferred to Eppendorf tubes and homogenized in buffer solutions [100 mM Tris, pH 7.4, $10 \mathrm{mM}$ EDTA, $1 \%$ sodium dodecyl sulfate (SDS), $100 \mathrm{mM} \mathrm{Na}_{2} \mathrm{P}_{2} \mathrm{O}_{7}, 100$ $\mathrm{mM} \mathrm{NaF}$, and $\left.10 \mathrm{mM} \mathrm{Na}_{2} \mathrm{VO}_{4}\right]$. Extracts were centrifuged at $12,000 \mathrm{rpm}$ for $40 \mathrm{~min}$ at $4{ }^{\circ} \mathrm{C}$ to remove the insoluble material. Protein content in the supernatant was quantified using the Bradford protein assay (BioRad Lab, Richmond, CA, USA) and treated with Laemmli buffer; $25-50 \mu \mathrm{g}$ of total protein was separated by polyacrylamide gel electrophoresis $(6.5 \%$ SDS-PAGE and $12 \%$ polyacrylamide) and transferred to $0.45-\mu \mathrm{m}$ nitrocellulose membranes. The membranes were blocked for $1 \mathrm{~h}$ in $5 \%$ non-fat milk. Subsequently, the samples were incubated overnight at $8{ }^{\circ} \mathrm{C}$ with antibodies against IR, IRSI, PI3K, Akt, pAkt (serine residue 473), and ERKl diluted in a blocking buffer containing $3 \%$ non-fat dry milk. They were then washed without milk for $30 \mathrm{~min}$ with the blocking buffer. Finally, the intensities of the bands were quantified by optical densitometry (ImageJ sofware; imagej.net/Downloads), and Ponceau $S$ staining was performed to obtain a loading control. All antibodies were purchased from Santa Cruz Biotechnology (Santa Cruz, Dalas, USA).

\section{Statistical analysis}

All results are expressed as mean \pm SEM. Two-way analysis of variance followed by Bonferroni's post hoc test was used to compare the two groups. Student's $t$-test was used whenever necessary. The level of significance was set at $p<0.05$. All statistics and graphs were analyzed using 
Prism software for Windows, version 5.01 (GraphPad Software, San Diego, CA, USA).

\section{Results}

\section{Body weight gain and wet weight/dry weight ratio}

The body weight of the rats at the beginning of the study was similar between the groups. At the end of the final protocol (after 10 days of treatment), the rats in the DEX group exhibited a body weight loss of $15.42 \%(p<0.05)$, whereas the rats in the CON group exhibited a body weight gain of $7.99 \%(p<0.05)$ (Fig. IA). The wet and dry weights (g) of masseter muscles did not statistically differ between the groups (Fig. IB and IC). The ratio between the weight of the masseter muscle and body weight was $16.87 \%$ higher in the DEX group than in the CON group (Fig. 1D).

Degree of expression of IR, IRSI, and PI3K and degree of phosphorylation/activity of IRSs and Akt

The degree of expression of IR, IRS1, and PI3K obtained from sections of the masseter muscle of the rats in the DEX group. The analysis of the intensity of bands in the membranes by densitometry showed that the levels of IR (Fig. 2A) and PI3K (Fig. 2B) in the masseter muscle were $56.85 \%$ and $30.73 \%$, respectively, lower in the DEX group than in the CON group $(p<0.05)$. The total level of IRSI was similar between the groups (Fig. 2C). Figure 2D shows the degree of phosphorylation/activity of IRS1 obtained from sections of the masseter muscle of rats in the DEX and CON groups at the beginning of the experiment [time zero $(-)]$ and after insulin stimulus $(+)$. After insulin stimulus, the degree of phosphorylation/activity of tyrosine residues of IRSI in the masseter muscle of the rats in the CON group was $38.15 \%$ higher than that at time zero (-). However, in the DEX group, there was no difference in the degree of phosphorylation before and after insulin stimulus (Fig. 2D). The total level of Akt in the masseter muscle (Fig. 2E) was similar between the groups. Figure $2 F$ shows the degree of phosphorylation/activity of Akt (phosphorylated at serine 473) obtained from sections of the masseter muscle of rats in the CON and DEX groups at time zero $(-)$ and after insulin stimulus $(+)$. After insulin stimulus, the degree of phosphorylation/activity of Akt from sections of the masseter muscle in the $\mathrm{CON}$ group was $513.18 \%$ higher than that at time zero (-). The DEX group also showed an increase in Akt phosphorylation compared with basal phosphorylation,
A

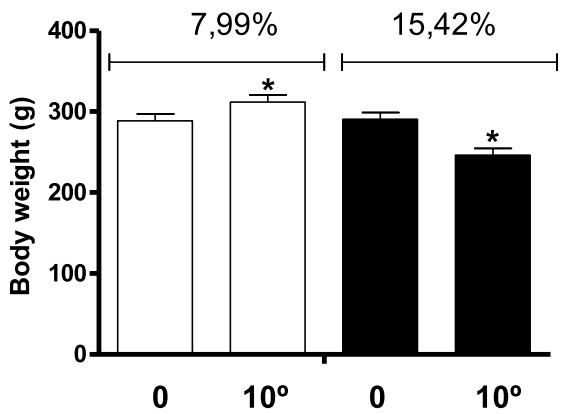

C

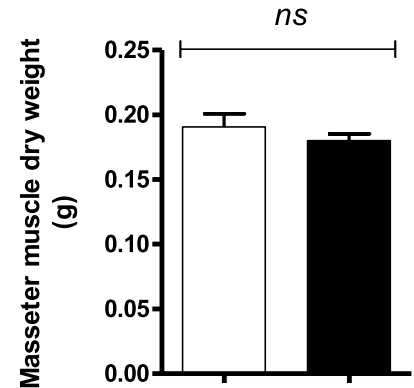

B

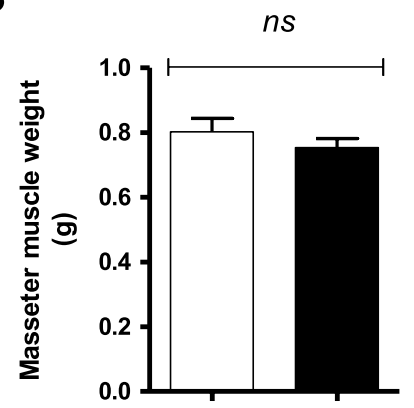

D

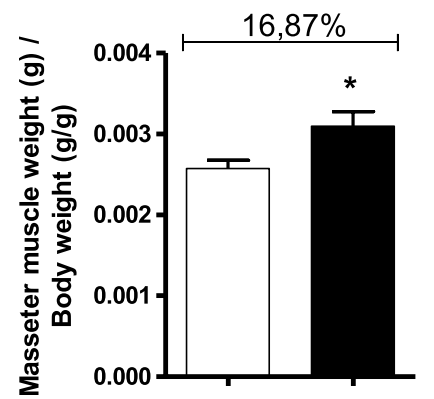

Fig. 1. Assessment of body weight and masseter muscle weight (masseter muscle weight and masseter muscle dry weight) in the CON (hollow bars; $n=10$ ) and DEX groups (solid bars; $n=10$ ). (A) Analysis of body weight in the CON and DEX groups at the beginning of dexamethasone treatment (time zero) and after 10 days of treatment. (B) Analysis of masseter muscle weight (g) in the CON and DEX groups after 10 days of treatment. (C) Analysis of muscle dry weight (g) in the CON and DEX groups after 10 days of treatment. (D) Ratio between the masseter muscle weight and body weight in the CON and DEX groups. Student's $t$-test was used for the analysis between the groups $\left({ }^{*} p<0.05\right)$ 
A
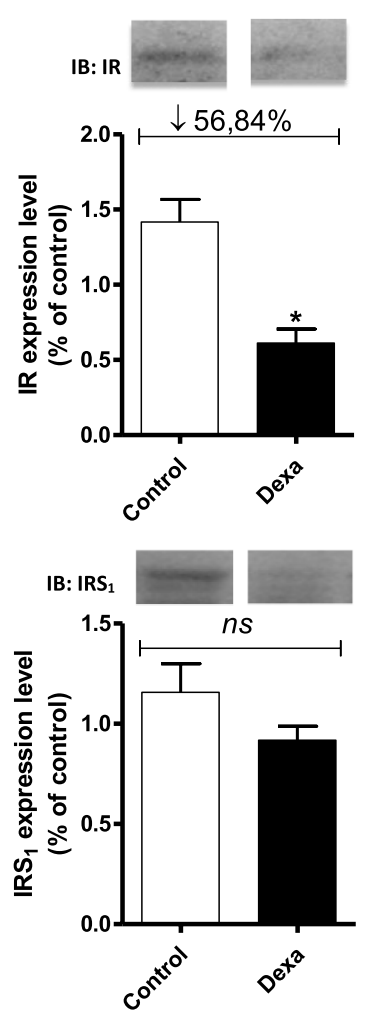

$\mathbf{E}$

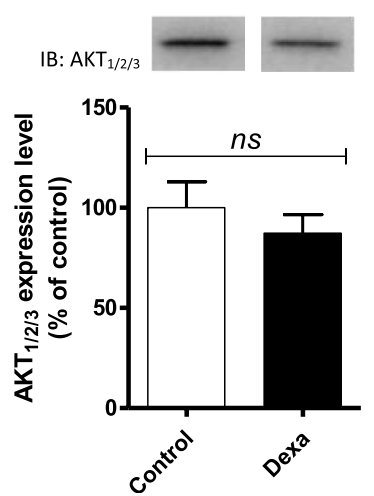

B

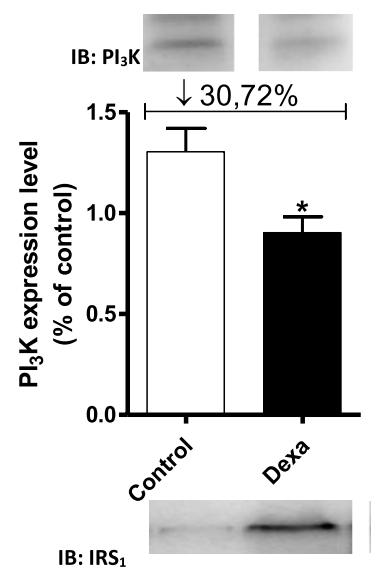

D

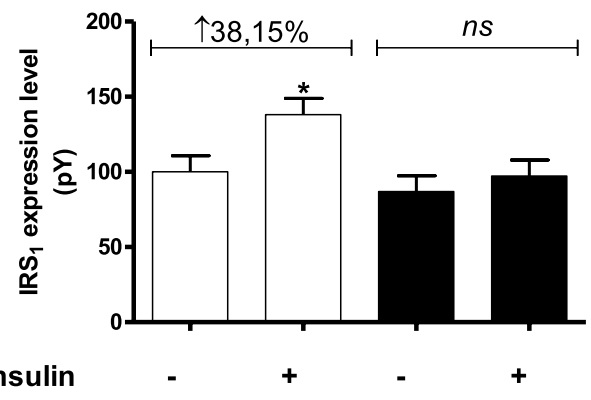

$\mathbf{F}$
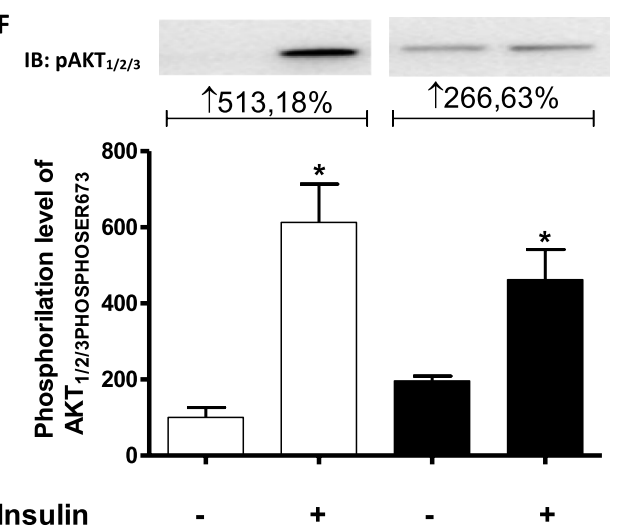

Fig. 2. Samples containing $25-50 \mathrm{mg}$ of solubilized proteins were subjected to SDS-PAGE and immunoblotting using specific antibodies. A blot representative of the experiments is shown. The status of phosphorylation and protein expression (percentage) involved in intracellular insulin signaling in the masseter muscle of rats in the CON (hollow bars; $n=6$ ) and DEX groups (solid bars; $n=6$ ) was determined by stoichiometry. Analysis of the degree of expression of IR (A), PI3K (B), and IRSI (C) in the masseter muscle in the CON and DEX groups. Analysis of the degree of IRS1 phosphorylation/activity in the masseter muscle in the CON and DEX groups before [time zero $(-)]$ and after the infusion of insulin into the portal vein $(+)(\mathrm{D})$. Total amount of Akt protein in the masseter muscle in the CON and DEX groups (E). Analysis of the degree of phosphorylation/activity (phosphorylation at serine 473) of Akt in the masseter muscle in the CON and DEX groups before [time zero $(-)]$ and after the infusion of insulin into the portal vein $(+)(\mathrm{F})$. Student's $t$-test was used in the intergroup analysis $\left({ }^{*} p<0.05\right)$

but the magnitude of this increase was attenuated after insulin stimulus (266.63\%) (Fig. 2F).

\section{Degree of ERKI expression}

The expression of ERKI obtained from sections of the masseter muscle of rats in the DEX and CON groups. The analysis of the intensity of bands in membranes by densitometry indicated significant differences between the groups (Fig. 3).

\section{Discussion}

In this study, treatment with dexamethasone induced body weight loss, which is in line with literature data 


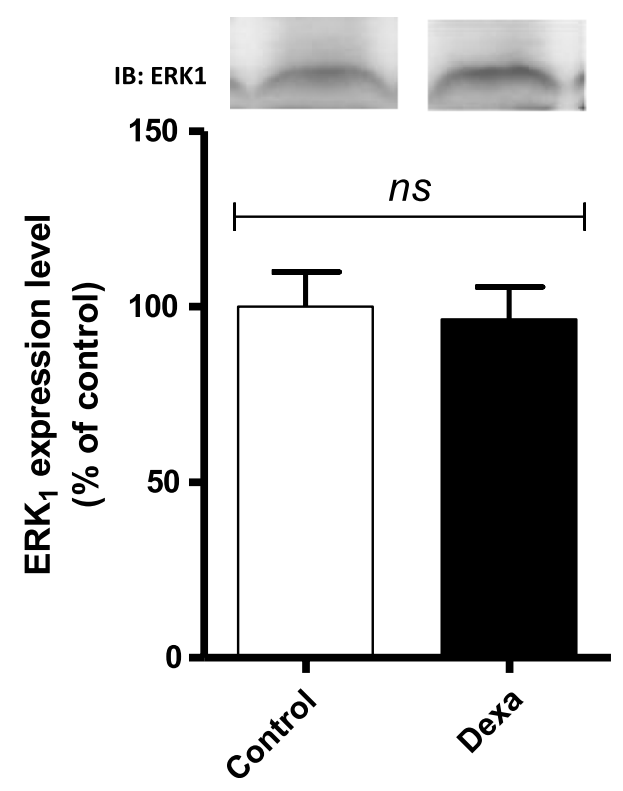

Fig. 3. Analysis of the degree of ERKl expression in the masseter muscle of rats in the CON (hollow bars; $n=6$ ) and DEX groups (solid bars; $n=6$ ). Student's $t$-test was used in the analysis between the groups $(p<0.05)$

associated with reduced food intake and dose-dependent weight gain [31]. Similar studies have demonstrated that treatment with dexamethasone at $1 \mathrm{mg} / \mathrm{kg}$ body weight leads to body weight loss, muscular atrophy, and resistance to insulin as shown in the anterior tibialis and extensor digitorum longus muscles [22]. However, this study did not show any significant difference in the wet and dry weights of the masseter muscle.

The ratio between masseter muscle weight and body weight was significantly higher in the DEX group. The studies have shown that muscular atrophy caused by high doses and/or the chronic use of dexamethasone is a consequence of a reduced rate of muscle protein synthesis and an increased rate of muscle proteolysis, which is a result of the impairment of several kinases of the insulin signaling pathway $[24,32]$.

Dexamethasone, which is a potent synthetic glucocorticoid analog of cortisol, was used to induce resistance to insulin. It is widely used in the field of pharmacology for its dose-dependent anti-inflammatory and immunosuppressive activities [19, 33]. Moreover, its chronic use induces adverse effects in the body, such as skeletal muscle myopathy $[22,23]$ and muscular weakness and deterioration in patients with Cushing's syndrome [24].

Few studies in the literature have investigated the insulin signaling pathway in the masseter muscle, which is a complex muscle that covers almost the entire side of the jaw and is thus involved in mastication, salivation, and speech. In male rats, masseter muscle fibers exhibit an intracellular response to the action of testosterone, which in turn promotes the phenotypic transformation of part of the slow fibers into fast fibers [34]. According to these authors, the predominance of fast fibers in this muscle appears to be associated with the increase in force generation during mastication.

In this study, total IR expression was lower in the DEX group than in the CON group, which may result in changes in the activity or degree of phosphorylation of intracellular proteins located downstream of the IR/IGFIR signaling pathway. Other researchers [35] reported that there was an increase in the degree of IRSI phosphorylation in primary cultures of skeletal muscle myocytes incubated with dexamethasone for $48 \mathrm{~h}$. Some authors [36] suggested that dexamethasone induces a decrease in the total content of IRSI was associated with an increase in the degree phosphorylation in muscles of the posterior limbs of rats. However, in our study in the masseter muscle, there was no significant difference in total IRSI content after treatment of dexamethasone.

There was an increase in the degree of phosphorylation/ activity of IRSI in the masseter muscle of the CON rats after insulin stimulus but not in the muscle of the rats treated with dexamethasone. Our results differed from those obtained by Saad et al. [36] who investigated the leg's hindlimb muscle. We do not exclude the hypothesis that proteins involved in the IR/IGFIR signaling pathway have distinct levels and/or degrees of phosphorylation, because they exhibit tissue-specific responses. Burén et al. [28] stated that the degree of phosphorylation of proteins involved in the IR/IGFlR signaling pathway isolated from fast muscle fibers is different from that of fibers in other muscle territories, such as the soleus muscle.

The masseter muscle of the rats treated with dexamethasone had a lower total PI3K level than that of the $\mathrm{CON}$ rats. PI3K is involved in the metabolism of glucose stimulated by insulin and acts on mitogenesis and cell differentiation [11, 37]. The phosphorylation of PI3K in turn increases the degree of phosphorylation of the serine residues of $\mathrm{PKB}$ (also known as Akt). Akt is an important central component of the signaling pathway and is involved in protein synthesis (via the activation of mammalian target of rapamycin kinase and of the glycogen synthase kinase $3 \beta$ pathway) $[32,38]$ and in intracellular protein degradation (mediated by the transcription of proteins belonging to the FoxO family) $[39,40]$.

Although there was no significant change in the expression of total Akt in this study, there was an increase in the degree of Akt phosphorylation in the CON group after insulin stimulus. The increase in the degree of phosphorylation was attenuated in the DEX group after insulin stimulus. According to Umeki et al. [23], treatment with dexamethasone for 2 weeks promotes atrophy of the masseter muscle and these events are related to the downregulation of the $\mathrm{Akt} / \mathrm{mTOR}$ pathway and upregulation of the proteolytic pathway (MURFl and ATROGIN). These effects are probably 
caused by the high glucocorticoid concentration that was used (six times higher than that used in this study).

Previs et al. [16] reported that the involvement of different isoforms of IRSs, e.g., IRS1 and IRS2, when phosphorylated, acts differently on different organs and on body development. They demonstrated that the deletion of IRSI in the body causes delayed growth and contributes to the development of resistance to insulin. The total deletion of IRS 2 induces the development of diabetes, beta-pancreatic cell dysfunction, and peripheral and hepatic resistance to insulin. A review conducted by Thirone et al. [17] on the ERK/MAPK pathway also showed that these substrates have distinct actions on the skeletal muscle. IRS2 appears to act directly on the Akt/ ERK/p38MAPK-dependent mitogenic and metabolic pathways, whereas IRSI is implicated in events leading to the translocation of vesicles containing GLUT4 to the plasma membrane and in glucose uptake. In this study, the total level of ERKI in the masseter muscle was similar between the groups; as no atrophy of the masseter muscle was observed, we suggest that the IRS2 pathway was unchanged.

In conclusion, treatment with dexamethasone promoted the reduction in the total amount of IR and PI3K. The degrees of phosphorylation of IRSI and Akt were altered without implications for the total weight of the masseter muscle. These results indicate that the masseter muscle, because it is composed of fast fibers, exhibits a degree of phosphorylation/activity that is different from that of fibers in other muscle territories. In addition, the role of other pathways, such as those mediated by IRS2/ Akt/ERK/p38MAPK, in the preservation of this muscle should not be ruled out.

$$
* * *
$$

Funding sources: This work was supported by Foundation for Research Support and Innovation in the State of Sergipe (FAPITEC), Coordination of Improvement of Higher Education Personnel (CAPES), and National Council for Scientific and Technological Development (CNPq).

Authors' contribution: FIRD, DM-S, GVM, FBL, CRDOC, and ACM: conception and design of the research. FIRD, DM-S, and GVM: acquisition of data, analysis and interpretation of the data, and writing of the manuscript. FIRD and DM-S: statistical analysis. FBL, CRDOC, and ACM: critical revision of the manuscript for intellectual content.

Conflict of interest: The authors declare no conflict of interest.

\section{References}

1. Kelly T, Yang W, Chen CS, Reynolds K, He J: Global burden of obesity in 2005 and projections to 2030. Int J Obesity 32, 1431-1437 (2008)

2. Sandouk Z, Lansang MC: Diabetes with obesity - Is there an ideal diet? Cleve Clin J Med 84, 4-14 (2017)
3. Braddon FEM, Rodgers B, Wadsworth MEJ, Davies JMC: Onset of obesity in a 36 year birth cohort study. BMJ 293, 299-303 (1986)

4. Guh DP, Zhang W, Bansback N, Amarsi Z, Birmingham L, Anis $\mathrm{AH}$ : The incidence of co-morbidities related to obesity and overweight: A systematic review and meta-analysis. BMC Public Health 9, 1-20 (2009)

5. Capehorn MS, Haslam DW, Welbourn R: Obesity treatment in the UK health system. Curr Obes Rep 5, 320-326 (2016)

6. DeFronzo RA, Ferrannini E: Insulin resistance: A multifaceted syndrome responsible for NIDDM, obesity, hypertension, dyslipidemia, and atherosclerotic cardiovascular disease. Diabetes Care 14, 173-194 (1991)

7. Rafacho A, Marroquí L, Taboga SR, Abrantes LF, Silveira LR, Boschero AC, Carneiro EM, Bosqueiro R, Nadal A, Quesada I: Glucocorticoids in vivo induce both insulin hypersecretion and enhanced glucose sensitivity of stimulus-secretion coupling in isolated rat islets. Endocrinology 151, 85-95 (2010)

8. DeFronzo RA, Ferrannini E, Groop L, Henry RR, Herman WH, Holst JJ, Hu FB, Kahn CR, Raz I, Shulman GI, Simonson DC, Testa MA, Weiss R: Type 2 diabetes mellitus. Nat Rev Dis Primers 1, 15019-15019 (2015)

9. Kim MK, Reaven GM, Kim SH: Dissecting the relationship between obesity and hyperinsulinemia: Role of insulin secretion and insulin clearance. Obesity 25, 378-383 (2016)

10. Velloso LA, Carneiro EM, Crepaldi SC, Boschero AC, Saad MJA: Glucose- and insulin-induced phosphorylation of the insulin receptor and its primary substrates IRS-1 and IRS-2 in rat pancreatic islets. FEBS Lett 377, 353-357 (1995)

11. Saltiel AR, Kahn CR: Insulin signalling and the regulation of glucose and lipid metabolism. Nature 414, 799-806 (2001)

12. Khan A, Pessin J: Insulin regulation of glucose uptake: A complex interplay of intracellular signalling pathways. Diabetologia 45, 1475-1483 (2002)

13. Kulkarni RN: Receptors for insulin and insulin-like growth factor-1 and insulin receptor substrate-1 mediate pathways that regulate islet function. Biochem Soc Trans 30, 317-322 (2002)

14. Houmard JA, Pories WJ, Dohm GL: Severe obesity: Evidence for a deranged metabolic program in skeletal muscle? Exerc Sport Sci Rev 40, 204-210 (2012)

15. Zhou X, Shentu P, Xu Y: Spatiotemporal regulators for insulinstimulated GLUT4 vesicle exocytosis. J Diabetes Res 2017, 1-9 (2017)

16. Previs SF, Withers DJ, Ren J, White MF, Shulman GI: Contrasting effects of IRS-1 versus IRS-2 gene disruption on carbohydrate and lipid metabolism in vivo. J Biol Chem 275, 38990-38994 (2000)

17. Thirone AC, Huang C, Klip A: Tissue-specific roles of IRS proteins in insulin signaling and glucose transport. Trends Endocrinol Metab 17, 72-78 (2006)

18. Gathercole LL, Morgan SA, Bujalska IJ, Hauton D, Stewart PM, Tomlinson JW: Regulation of lipogenesis by glucocorticoids and insulin in human adipose tissue. PLoS One 6, 26223-26231 (2011)

19. Strehl C, Buttgereit F: Optimized glucocorticoid therapy: Teaching old drugs new tricks. Mol Cell Endocrinol 380, 32-40 (2013)

20. Christ-Crain M, Kola B, Lolli F, Fekete C, Seboek D, Wittmann G, Feltrin D, Igreja SC, Ajodha S, Harvey-White J, Kunos G, Müller B, Pralong F, Aubert G, Arnaldi G, Giacchetti G, Boscaro M, Grossman AB, Korbonits M: AMP-activated protein kinase mediates glucocorticoid-induced metabolic changes: A novel mechanism in Cushing's syndrome. Faseb J 22, 1672-1683 (2008)

21. Van Raalte DH, Ouwens DM, Diamant M: Novel insights into glucocorticoid-mediated diabetogenic effects: Towards expansion of therapeutic options? Eur J Clin Investig 39, 81-93 (2009)

22. Barel M, Perez OAB, Giozzet VA, Rafacho A, Bosqueiro JR, Amaral SL: Exercise training prevents hyperinsulinemia, muscular glycogen loss and muscle atrophy induced by dexamethasone treatment. Eur J Appl Physiol 108, 999-1007 (2009) 
23. Umeki D, Ohnuki Y, Matotani Y, Shiozawa K, Suita K, Fukita T, Nakamura Y, Saeki Y, Okumura S: Protective effects of clenbuterol against dexamethasone-induced masseter muscle atrophy and myosin heavy chain transition. PLoS One 10, e0128263 (2015)

24. Tsuchida W, Iwata M, Akimoto T, Matsuo S, Asai Y: Heat stress modulates both anabolic and catabolic signaling pathways preventing dexamethasone-induced muscle atrophy in vitro. J Cellul Physiol 232, 650-664 (2016)

25. Ruzzin J, Wagman AS, Jensen J: Glucocorticoid-induced insulin resistance in skeletal muscles: Defects in insulin signalling and the effects of a selective glycogen synthase kinase-3 inhibitor. Diabetologia 48, 2119-2130 (2005)

26. Gholap S, Kar A: Gymnemic acids from Gymnema sylvestre. Potentially regulates dexamethasone-induced hyperglycemia in mice. Pharm Biol 43, 192-195 (2005)

27. Anhe GF, Hirabara SM, Turrer TC, Caperuto LC, Anhe FF, Ribeiro LM, Marçal AC, Carvalho CR, Curi R, Machado UF, Borfin S: Postpartum glycemic homeostasis in early lactating rats is accompanied by transient and specific increase of soleus insulin response through IRS2/AKT pathway. Am J Physiol Regul Integ Comp Physiol 292, 2225-2233 (2007)

28. Burén J, Lai YC, Lundgren M, Eriksson JW, Jensen J: Insulin action and signalling in fat and muscle from dexamethasone-treated rats. Archiv Biochem Biophys 474, 91-101 (2008)

29. Okamoto MM, Anhê GF, Sabino-Silva R, Marques MFDSF, Freitas HS, Mori RCT, Melo KFS: Intensive insulin treatment induces insulin resistance in diabetic rats by impairing glucose metabolismrelated mechanisms in muscle and liver. J Endocrinol 211, 55-64 (2011)

30. DeFronzo RA, Tripathy D: Skeletal muscle insulin resistance is the primary defect in type 2 diabetes. Diabetes Care 32, 157-163 (2009)
31. Jahng JW, Kim NY, Ryu V, Yoo SB, Kim BT, Kang DW, Lee JH: Dexamethasone reduces food intake, weight gain and the hypothalamic 5-HT concentration and increases plasma leptin in rats. Eur J Pharmacol 581, 64-70 (2008)

32. Egerman MA, Glass DJ: Signaling pathways controlling skeletal muscle mass. Crit Rev Biochem Mol Biol 49, 59-68 (2013)

33. Pasieka A, Rafacho A: Impact of glucocorticoid excess on glucose tolerance: Clinical and preclinical evidence. Metabolites 6, 24 (2016)

34. Widmer CG, English AW, Morris-Wiman J: Developmental and functional considerations of masseter muscle partitioning. Archiv Oral Biol 52, 305-308 (2007)

35. Gathercole LL, Bujalska IJ, Stewart PM, Tomlinson JW: Glucocorticoid modulation of insulin signaling in human subcutaneous adipose tissue. J Clin Endocrinol Metab 92, 4332-4339 (2007)

36. Saad MJA, Folli F, Kahn JA, Kahn CR: Modulation of insulin receptor, insulin receptor substrate-1, and phosphatidylinositol 3 -kinase in liver and muscle of dexamethasone-treated rats. J Clin Investig 92, 2065-2072 (1993)

37. Glass DJ: PI3 kinase regulation of skeletal muscle hypertrophy and atrophy. Curr Top Microb Immunol 1, 267-278 (2010)

38. Schiaffino S, Mammucari C: Regulation of skeletal muscle growth by the IGFl-Akt/PKB pathway: Insights from genetic models. Skel Musc 1, 4-18 (2011)

39. Léger B, Cartoni R, Praz M, Lamon S, Dériaz O, Crettenand A, Gobeleti C, Rohmer P, Konzelmann M, Luthi F, Russell AP: Akt signalling through GSK-3 $\beta$, mTOR and Foxol is involved in human skeletal muscle hypertrophy and atrophy. J Physiol 576, 923-933 (2006)

40. Bacurau AV, Cunha TF, Souza RW, Voltarelli VA, Gabriel-Costa D, Brum PC: Aerobic exercise and pharmacological therapies for skeletal myopathy in heart failure: Similarities and differences. Oxid Med Cell Longev 2016, 1-16 (2016) 